\title{
Numerical Computations in the Worldsheet Formulation
}

\author{
H. Fort \\ Instituto de Física, Facultad de Ciencias, Tristan Narvaja 1674, 11200 Montevideo, Uruguay*
}

\begin{abstract}
The worldsheet formulation of lattice gauge theories has two appealing features: the gauge non-redundancy and the geometrical transparency. Both properties are profitable in order to perform numerical computations. In the case of dynamical fermions this description offers additional advantages. For instance, it does not suffer from the species doubling problem and it involves fewer degrees of freedom.
\end{abstract}

\section{The Worldsheet Formulation Approach}

Simulating QCD with dynamical fermions or full QCD is still too expensive in computer time. Year after year the conclusion at lattice meetings is the same: either more machine power and/or a real improvement in algorithms are needed to produce reliable estimates of hadron spectroscopy [1]. We propose to explore a new numerical approach based on the socalled $P$-representation [2]. Staggered fermions can be naturally introduced by considering open paths representing string-like "electromeson" configurations in addition to the closed ones or loops representing pure electric flux excitations. This gives rise to the space of paths $\mathrm{P}$ of the above mentioned P-representation [2]. All the physical operators have a geometrically transparent realization in this space.

The worldsheet formulation of lattice gauge theories (in terms of variables attached to the worldsheets of P-paths) was introduced for several models [3]-[5]. U(1) theory [3] and scalar electrodynamics $\left(\mathrm{SQED}_{c}\right)$ 㸃 were simulated using this representation and the results were quite encouraging. More recently, a worldsheet description of gauge theories with dynamical fermions was proposed [6].

The worldsheet partition function $Z_{P}$ of QED is expressed as a sum over the worldsheets of strings or paths of the P-representation. That is, surfaces such that: (I) their borders $\mathcal{F}^{c}$ are self-avoiding polymer-like loops and (II) when intersected with a time $t=$ constant plane they produce paths begining at even sites and end-

\footnotetext{
${ }^{*}$ Supported in part by CONICYT, Project No. 318.
}

ing at odd ones. This description differs from that one gets by integrating the fields in the Kogut-Susskind action in two features. In first place, the isolated links traversed in both opposite directions or "null" links were ruled out and with them the myriad of different configurations corresponding to a given configuration of worldsheets. In second place, by virtue of the constraint (II), the surfaces when intersected with a time $t=$ constant plane produce only paths begining at even sites and ending at odd ones instead of the paths of ends with whatever parity. This two differences translate respectively in: computer time economy and a cure of the species doubling problem of the Kogut-Susskind action.

The expression of $Z_{P}$ is as follows [8]:

$Z_{P}=\sum_{S_{\mathcal{F}^{c}}} \sigma\left(\mathcal{F}^{c}\right) \exp \left\{-\frac{1}{2 \beta} \sum_{p \in S_{\mathcal{F}^{c}}} n_{p}^{2}\right\}$,

where the sign $\sigma_{\mathcal{F}}$ is given in terms of purely geometric quantities of the fermionic loops $\mathcal{F}^{c}$ [6]. In ref.[6] it was proved that $Z_{P}$ leads to the QED Hamiltonian using the transfer matrix procedure.

Our aim was to test the worldsheet formulation of ref. [6] as a method to simulate dynamical fermions. Hence, we chose the simplest lattice gauge theory with fermions, the Schwinger model or $(1+1)$ QED, and we performed a Monte Carlo simulation. This massless model can be exactly solved in the continuum. However, it is rich enough to share many features with 4dimensional QCD as the confinnement and the breaking of the chiral symmetry with an axial anomaly. For this reason it has been extensively used as a laboratory to analyze the previous phe- 
nomena and also to test different techniques to simulate theories with fermions.

\section{The Schwinger Model in the Worldsheet formulation}

In $D=2$, it turns out that $\sigma\left(\mathcal{F}^{c}\right)$ can be expressed in terms of the number of connected parts $N_{\mathcal{F}^{c}}$, the lenght $L_{\mathcal{F}^{c}}$ and the area $A_{\mathcal{F}^{c}}$ of $\mathcal{F}^{c}$ as $\sigma\left(\mathcal{F}^{c}\right)=(-1)^{N_{\mathcal{F}^{c}}-\frac{L_{\mathcal{F}} c}{2}+A_{\mathcal{F}^{c}}}$ and all the nonvanishing contributions have $\sigma_{\mathcal{F}}=+1$. The reason is that $N_{\mathcal{F}^{c}}-\frac{L_{\mathcal{F}^{c}}}{2}+A_{\mathcal{F}^{c}}=I_{\mathcal{F}}^{c}$, the number of enclosed sites by the fermionic loops $\mathcal{F}^{c}$ which is always even by virtue of the above constraints (I) and (II) ( see ref.[6] for more details), so that we omit this factor. The fermionic paths $\mathcal{F}^{c}$ can be expressed in terms of integer 1-forms -attached to the links $-f$ with three possible values: 0 and \pm 1 with the constraint that they are non self-crossing and closed $(\partial f=0)$ as

$Z_{P}^{\text {Schwinger }}=\sum_{n} \sum_{f} \exp \left\{-\frac{1}{2 \beta}\|n\|^{2}\right\} \delta(f-\partial n) \cdot(2)$

The lattice chiral condensate per-lattice-site is defined as $<\bar{\chi} \chi>=\frac{1}{2 N_{s}} \sum_{x}(-1)^{x_{1}}<$ $\left[\hat{\chi}^{\dagger}(\mathrm{x}), \hat{\chi}(\mathrm{x})\right]>$, where $N_{s}$ is the number of lattice sites. The corresponding operator is realized in the P-representation and thus we get for the chiral condensate [2]:

$$
<\bar{\chi} \chi>=\frac{1}{2}-\frac{2 \mathcal{N}_{P}}{N_{s}},
$$

where $\mathcal{N}_{P}$ is the number of connected paths at a given time $t$. Thus, equation (3) allows to calculate directly the chiral condensate simply by counting the number of "electromesons" we have when we intersect their world sheets with each time slice $t$.

\section{Monte Carlo Simulation and Results}

To generate the worldsheets of the string-like configurations we use a Metropolis-type updating algorithm with the Boltzman weight proportional to $\exp \left\{-\frac{1}{2 \beta} \sum_{p} n_{p}^{2}\right\}$. We simulate the model on $L \times L$ square lattices with periodic boundary conditions $(p b c)$.
All the plaquettes $p$ belonging to an open surface, by virtue of the self-avoiding constraint on their borders, must have all their plaquettes fulfilling the condition that the difference between integers $n_{p}$ of contiguous plaquettes has 3 possibilities: $\Delta n_{p}= \pm 1$ or 0 . There are also closed surfaces corresponding to the world sheets of looplike pure electric flux lines. In two dimensions the only possibility for a pure electric (closed) surface is to cover the entire lattice and then being closed by the $p b c$. Thus, all the plaquettes $p$ belonging to it have assigned the same integer value of $n_{p}= \pm 1, \pm 2, \ldots$. Our algorithm works as follows: whenever there are different possible values for the integer $n_{p}$ attached to plaquette $p$ - such that the produced surface fulfills the constraints (I) and (II) (self avoiding frontiers and odd length string respectively) - the present value is changed to one of these values and the Metropolis test is performed. Sometimes there is only one possible value of $n_{p}$ consistent with the constraints (I) and (II), in such cases the $n_{p}$ is updated to it and the Metropolis test is skipped. Typically, 100000 sweeps per point were performed.

To compute $<\bar{\chi} \chi>$ using (3) we have to count $\mathcal{N}_{P}$ at each one of the $L$ time slices. Therefore for each lattice sweep we collect $L$ values of $\langle\bar{\chi} \chi>$.

First, we checked that we get the right strong coupling behavior both for the ground-state energy density $\omega_{0}$ and the chiral condensate perlattice-site $\langle\bar{\chi} \chi>$. The agreement with the series expansion is pretty well up to $\beta=0.12$ as one can see from FIG.1.

On the other hand, the continuum theory is reached at zero lattice coupling, in the same way as four dimensional asymptotically free theories like QCD. It is known exactly that the chiral condensate in the continuum is given by

$\frac{<\bar{\psi} \psi>}{e}=\frac{e^{\gamma}}{2 \pi^{3 / 2}}=0.15995$.

As long as the lattice size $L$ is increased, the convergence of $q=<\bar{\chi} \chi>/ e$ to its known continuum value (何) improves. A property of the action of (2) is that $q$ becomes stabilized at its continuum value well inside the weak coupling. Before reaching this regime, this observable oscillates strongly with $\beta$. In FIG. 2 we plot $q$ vs. $\beta$ 


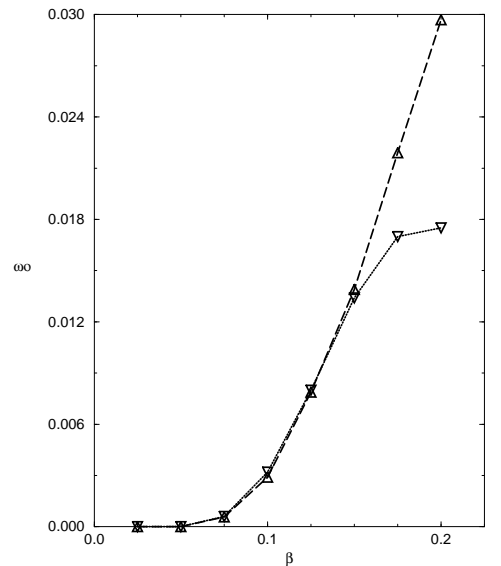

Figure 1. (a) The strong coupling behavior of $\omega_{o}$ on a $4 \times 4$ lattice (up triangles). (b) The corresponding strong coupling series (down triangles).

for lattices of $16 \times 16,20 \times 20,24 \times 24$ and $28 \times 28$. The value at which the simulation stabilizes at the weak coupling regime decreases from around 0.29 for $L=16$ to 0.17 for $L=28$.

\section{Conclusions and Final Remarks}

Our proposal was to show that the recently introduced worldsheet formulation was a valuable and alternative tool in order to do numerical computations with dynamical fermions. The method present the following advantages: 1) The easiness of computation. 2) Economy I: no gauge redundancy. 3 Economy II: it involves fewer degrees of freedom that the Kogut-Susskind action. 4) As a consequence of the constraint (II), the worldsheet action does not suffer from the species doubling problem of the Kogut-Susskind action.

The values for the chiral condensate are consistent with the exact results for the continuum theory (recovered at the weak coupling fixed point). In addition, our strong coupling results are in agreement with the strong coupling expansion.

\section{REFERENCES}

1. QCD-TARO Collaboration, Lattice 96, Nucl. Phys. B53 (Proc. Suppl.) (1997) 938; Philippe de Forcrand, Lattice 95 Published in

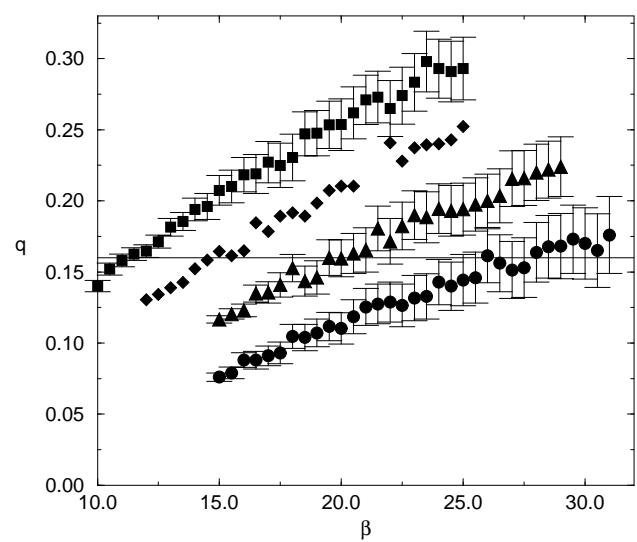

Figure 2. $q=<\bar{\chi} \chi>/ e$ for $L=16$ (squares), $L=$ 20 (diamonds), $L=24$ (triangles) and $L=28$ (circles). The horizontal line correspond to the continuum limit value $\simeq 0.16$.

Lattice 1995 (1996) 228; C. Michael, Lattice 94, Nucl. Phys. B42 (Proc. Suppl.) (1995); D. Weingarten, Lattice 93, Nucl. Phys. B34 (Proc. Suppl.) (1994) 29; A. Ukawa, Lattice 92, Nucl. Phys. B30 (Proc. Suppl.) (1993) 3 ;D. Toussaint, Lattice 91, Nucl. Phys. B26 (Proc. Suppl.) (1992) 3; T.A. De Grand Lattice 90, Nucl. Phys. B20 (Proc. Suppl.) (1991)

2. H. Fort and R.Gambini, Phys.Rev. D44 (1991) 1257.

3. J.M. Aroca, M. Baig and H. Fort, Phys. Lett. B336 (1994) 54.

4. J.M. Aroca, M. Baig, H. Fort and R. Siri, Phys. Lett. B366 (1995) 416.

5. J.M. Aroca, H. Fort and R. Gambini, heplat/9703007

6. J.M. Aroca, H. Fort and R. Gambini, heplat/9607050 (July 1996).

7. L. Susskind, Phys.Rev. D16 (1977) 3031; Banks T., D.R.T. Jones, J. Kogut, S. Raby, P.N. Scharbach, D.K. Sinclair and L. Susskind, Phys.Rev. D15 (1977) 1111.

8. This version of $Z_{P}$ corresponds to the Villain form of the lattice action (J. Villain, J. Phys. (Paris) 36 (1975) 581). We chose this action only for simplicity. 